Check for updates

Cite this: Chem. Commun., 2019, 55, 10076

Received 30th May 2019,

Accepted 6th July 2019

DOI: $10.1039 / \mathrm{c} 9 \mathrm{cc} 04166 \mathrm{~d}$

rsc.li/chemcomm

\section{Self curing and voltage activated catechol adhesives $\uparrow$}

\author{
Lu Gan, $\ddagger^{\mathrm{a}}$ Nigel C. S. Tan, $\ddagger^{\mathrm{a}}$ Avi Gupta, ${ }^{\mathrm{b}}$ Manisha Singh, ${ }^{\text {ac }}$ Oleksandr Pokholenko, ${ }^{a}$ \\ Animesh Ghosh, ${ }^{a}$ Zhonghan Zhang, ${ }^{a}$ Shuzhou Li (D) ${ }^{a}$ and Terry W. J. Steele (DD ${ }^{\star a}$
}

\begin{abstract}
Catechol adhesives are limited to two-part curing designs. For the first time, a one-pot catechol adhesive is demonstrated with multiple modes of external activation: self-curing, electrocuring, substrate, and two-part curing. Lap shear adhesion (50 $\mathrm{kPa}$ ) and viscoelastic properties demonstrate that electrocuring is non-inferior to twopart curing methods.
\end{abstract}

Catechol-grafted polymers/conjugates are promising hydrogels towards bonding of biomaterials on a variety of surfaces. ${ }^{1-3}$ When catechol is oxidized to $o$-quinone, the latter covalently reacts with amines or itself by a number of mechanisms. ${ }^{4-6}$ Although chemical and chelate-mediated crosslinking provides rapid gelation, it requires adding additional initiators that need to be thoroughly mixed. Oxidants, ${ }^{7,8}$ chelating metal ions, ${ }^{8}$ and enzymes ${ }^{9}$ have been demonstrated to initiate the catechol crosslinking, where all fall under the designation of two-part chemical curing (Fig. 1a). Two-part curing has the advantage of rapid gelation, but this suffers from limited manipulation before application, local cytotoxicity, and narrow pH ranges. ${ }^{6,10}$ Thus, an unmet need exists for a one-pot catechol-mediated adhesive that allows self-curing (with a known lag-time) or through an external stimulus.

Low voltage initiation of Voltaglue bioadhesives has recently been demonstrated on matrices that incorporate donor/acceptor pairs. ${ }^{11,12}$ However, these first proof-of-concept Voltaglue designs required activation of diazirine, whose voltage activation occurs in the range of water electrolysis $(-1.6 \mathrm{~V}$ vs. $-1.23 \mathrm{~V}$, respectively)-imparting significant foaming into the matrix from $\mathrm{H}_{2}$ and $\mathrm{O}_{2}$ evolution. ${ }^{11}$ Catechols, in comparison, are

\footnotetext{
${ }^{a}$ School of Materials Science and Engineering (MSE), Division of Materials Technology, Nanyang Technological University (NTU), Singapore 639798. E-mail: wjsteele@ntu.edu.sg

${ }^{b}$ Department of Materials Science and Engineering, Indian Institute of Technology, Kanpur, India

${ }^{c}$ NTU-Northwestern Institute for Nanomedicine (NNIN), Interdisciplinary Graduate School (IGS), Nanyang Technological University (NTU), Singapore 637553

$\dagger$ Electronic supplementary information (ESI) available. See DOI: 10.1039/c9cc04166d

\$ These authors contributed equally to the work.
}

electrochemically activated well below water electrolysis. Considering the known advantages of catechol redox properties, ${ }^{13}$ a design of donor/acceptor pair forms our central hypothesis: dendrimer conjugates that graft catechols as electron/proton donor(s) paired with a reducible acceptor(s) will be voltage activated for quinone-mediated crosslinking and adhesion. This design would elevate catechols to one-pot adhesives with material properties that are dependent on electric external stimuli.

Previous work on voltage activated adhesives suggests that donor/acceptor pairs mediate electron transduction, even in a non-aqueous matrix. ${ }^{12}$ Catechol is oxidized to reactive intermediates, $o$-quinone, which are responsible for intermolecular crosslinking. Several catechol small molecules are available with

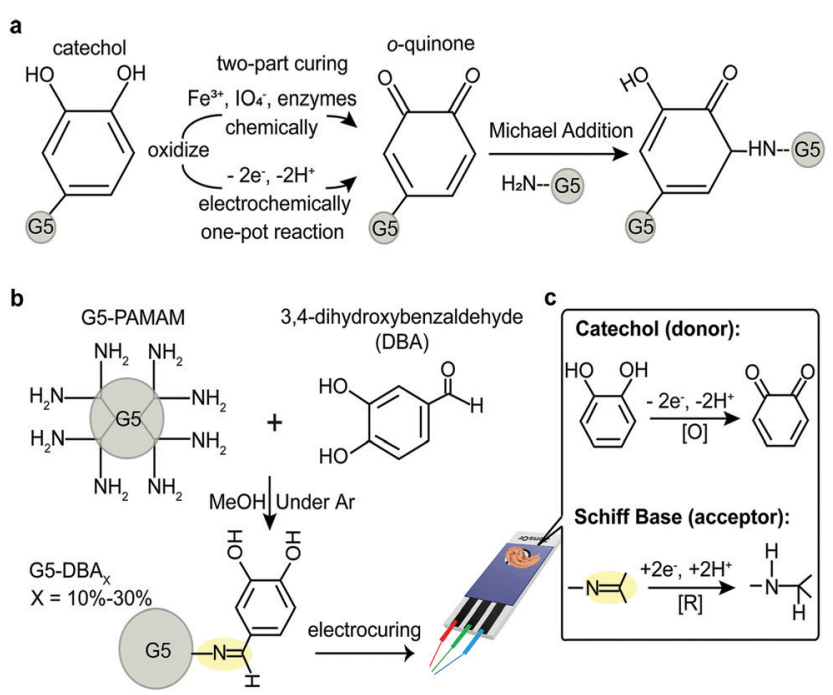

Fig. 1 (a) Schematic diagram of catechol being chemically oxidized or electrochemically oxidized into o-quinone, which is responsible to irreversible covalent crosslinking via Michael addition, for example. (b) Synthetic route of 3,4-dihydroxybenzaldehyde (DBA) grafted G5-PAMAM conjugates (G5-DBA $x$ ), with the grafting ratio $X=10 \%, 20 \%$, and $30 \%$, respectively. (c) Schematic illustration of plausible intramolecular donor/acceptor pair in G5-DBA $x$ conjugates, which effects the electrocuring performance. 
handles for dendrimer grafting. A strategy to simultaneously graft catechol (donor) and an acceptor group would reduce the synthesis to a one-pot reaction. Protocatechuic aldehyde or 3,4dihydroxybenzaldehyde (DBA) is a naturally derived catechol that spontaneously forms Schiff bases (azomethines) in the presence of amines. Schiff bases are regularly exploited for grafting and are rapidly reduced under aqueous conditions with mild reducing agents (e.g., sodium borohydride in SI Results, ESI $\dagger$ ). ${ }^{14,15}$ Simple mixing of DBA with G5-PAMAM (Fig. 1b) grafts both the donor (catechol) and the acceptor (Schiff base) in flawless 1:1 molar ratios to form zwitterionic tautomers that are thermodynamically stable (Fig. S1 and S2, ESI $†$ ). G5-PAMAM is utilized as the polymer macromolecule, as it offers numerous design advantages. ${ }^{11,12,16,17}$ The surface primary amines on the spherical dendrimer limit intramolecular crosslinking while preventing linear entanglements. ${ }^{18,19}$ It is also soluble in both aqueous and organic solvents, unlike chitosan macromers. PAMAM branched polymer is a model system for exploring structure activity relationships while maintaining an excess of amines for quinone-mediated crosslinking-further modifications are necessary for bioadhesive or underwater applications. Grafting ratios of $10-30 \%$ catechol conjugates are explored (G5-DBA ${ }_{X}$ with $X=10 \%, 20 \%, 30 \%$, respectively), but ratios $>40 \%$ had limited aqueous solubility (data not shown).

Reaction efficiency and final grafting ratios are assessed by ${ }^{1} \mathrm{H}$ NMR (Fig. S3-S6 and Tables S1, S2, ESI $\dagger$ ) and size exclusion chromatography (Fig. S7 and Table S2, ESI $\dagger$ ). Generation of the Schiff base is complete after $8 \mathrm{~h}$ as assessed by UV/vis at $405 \mathrm{~nm}$ (Fig. S7, ESI $\dagger$ ). This peak is extinguished after borohydride reduction, which removes the Schiff base acceptor as a control (Fig. S8, ESI $\dagger$ ). Molar mass of G5-DBA X $_{X}$ positively correlates with the grafting ratio with a reduction of the UV/RI peak elution volume (Fig. S7e, ESI $\dagger$ ). Redox properties of G5-DBA ${ }_{X}$ conjugates are examined by cyclic voltammetry (CV) in isotonic PBS electrolyte (Fig. 2a and Fig. S9, ESI $\dagger$ ). A catechol-free benzaldehyde is also grafted on G5-PAMAM to serve as a catechol-free control (G5-Benz $z_{20}$, Fig. S10, ESI $\dagger$ ), and borohydride reduced G5-DBA 20 serves as Schiff base free control (reduced G5-DBA 20 , Fig. S8, ESI $\dagger$ ). Free DBA (Fig. S9a, ESI $\dagger$ ) exhibits an irreversible and diffusion-controlled redox behavior, ${ }^{20}$ supported by the following observations: (1) the peak current ratio $\left(I_{\mathrm{pao}}^{E} / I_{\mathrm{pc} 0}^{E}\right)$ is less than 1 at the scan rate of $50 \mathrm{mV} \mathrm{s}^{-1}$; (2) both the anodic peaks $\left(E_{\mathrm{pa} 0}=0.52 \mathrm{~V}\right)$ and the cathodic peaks $\left(E_{\mathrm{pc} 0}=-0.02 \mathrm{~V}\right)$ (vs. $\mathrm{Ag} / \mathrm{AgCl}$ ) increase progressively with the increasing scan rates $(\nu)$; (3) the plot of peak current as a function of $\nu^{1 / 2}$ is linear for both peak $E_{\mathrm{pa} 0}$ and $E_{\mathrm{pco}}$ (Fig. S9b, ESI $\dagger$ ). The successive $\mathrm{CV}$ cycles (either use glassy carbon or Pt as working electrode) exhibited non-repeatable current signal (Fig. S9c and $\mathrm{d}$, ESI $\dagger$ ), suggesting that the electrode surface is fouled by electropolymerized DBA film, which in turn inactivates the electrode surface sensitivity. ${ }^{21,22}$ At the scan rate of $50 \mathrm{mV} \mathrm{s}^{-1}$, the cyclic voltammograms of $\mathrm{G} 5-\mathrm{DBA}_{10}$ retains the irreversible redox behavior of DBA. G5-DBA ${ }_{10}$ displays two anodic peaks $\left(E_{\mathrm{pa} 1}=0.29 \mathrm{~V}\right.$ and $\left.E_{\mathrm{pa} 2}=0.91 \mathrm{~V}\right)$ and a minute cathodic peak (Fig. 2a). Scan rate dependent CV confirms the existence of the $E_{\mathrm{pa} 2}$ peak (Fig. S9e, ESI $\dagger$ ). The origin of $E_{\mathrm{pa} 2}$ peak is attributed to the aldehyde/Schiff base, as it is observed in free DBA,
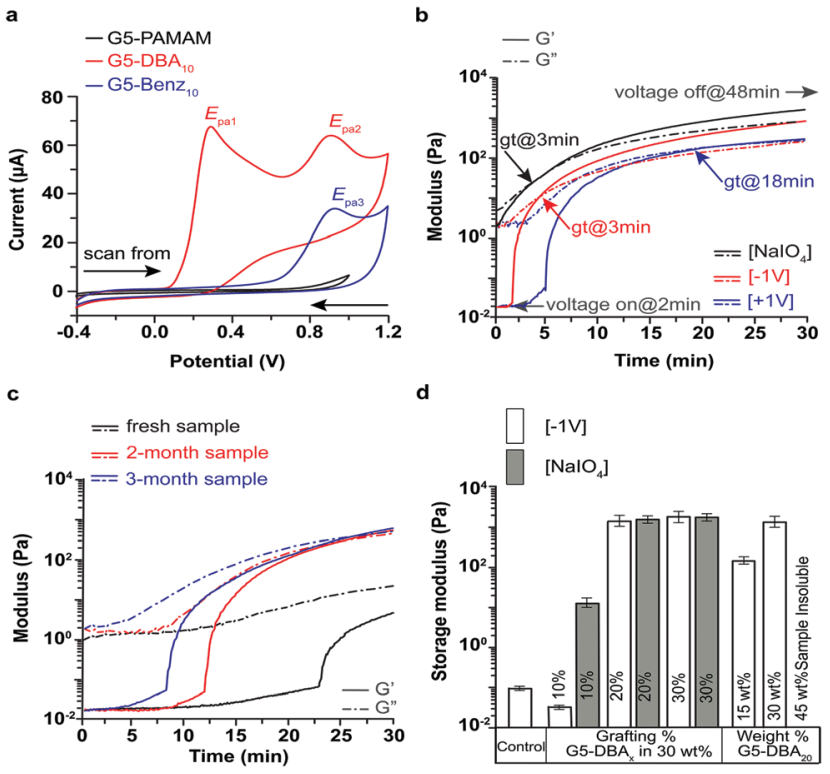

Fig. 2 (a) Cyclic voltammograms of G5-PAMAM (as background), G5-Benz 10 (as reference), and G5-DBA 10 at $50 \mathrm{mV} \mathrm{s}^{-1}$. Electrolyte: PBS (pH 7.2), working electrode: glassy carbon, reference electrode: $\mathrm{Ag} / \mathrm{AgCl}$, counter electrode: Pt. Real time dynamic mechanical analysis of formulation of $30 \mathrm{wt} \% \mathrm{G} 5-\mathrm{DBA}_{20}$ conjugates in PBS electrolyte: (b) two-part curing (mixed with $0.1 \mathrm{mM} \mathrm{NalO}_{4}$ solution) and electrocuring (voltage off: 0-2 min; voltage on: 2-48 min, throughout) at $\pm 1 \mathrm{~V}$, gt-gelation time. (c) self-curing (no voltage applied and no oxidant added) after rehydration. Storage modulus ( $G^{\prime}$, solid line) and loss modulus ( $G^{\prime \prime}$, dot dashed line). (d) $G^{\prime}$ values of G5-PAMAM (control) and $30 \mathrm{wt} \% \mathrm{G} 5-\mathrm{DBA}$ formulation as function of grafting $\%$ initiated by $-1 \mathrm{~V}$ voltage or $\mathrm{NaIO}_{4}$ oxidant and $\mathrm{G} 5-\mathrm{DBA}_{20}$ formulation as function of weight $\%$. $45 \mathrm{wt} \%$ formulation is insoluble in PBS. White columns-electrocuring at $-1 \mathrm{~V}$, grey columns-two-part curing with periodate.

G5-Ben $_{10}\left(E_{\mathrm{pa} 3}\right.$ in Fig. S9f, ESI $\left.\dagger\right)$, G5-DBA 10 , but not in G5PAMAM (Fig. 2a). All scans of G5-Ben ${ }_{10}$ are plotted the first cycle (Fig. S9e and f, ESI $\dagger$ ) since the peak current decreases after the second cycle. $E_{\mathrm{pa} 2}$ results from the known redox activation of Schiff bases under similar conditions. ${ }^{23-27}$

Real-time rheology is applied to evaluate the mechanical properties (storage modulus- $G^{\prime}$ and loss modulus- $G^{\prime \prime}$ ) before and after curing stimuli. The customized rheology platform incorporates a ceramic probe to avoid catechol-metal chelates, as aluminum probes/surfaces are found to instantly initiate curing (Fig. S10a, ESI $\dagger$ ). Voltages of $+1 \mathrm{~V}$ and $-1 \mathrm{~V}$ effects on

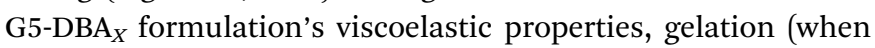
$G^{\prime}=G^{\prime \prime}$ ), and lag time (period before gelation) are compared to a typical two-part curing method (Fig. 2b). Two-part curing with periodate on $\mathrm{G} 5-\mathrm{DBA}_{20}$ achieves gelation within $3 \mathrm{~min}$ and exhibits a kPa shear modulus after 25 minutes. Negative voltage (cathode as WE) displays instantaneous initiation (Fig. 2b), which is removed after reduction of Schiff base (Fig. S8c, ESI $\dagger$ ). Voltage initiation rapidly increases $G^{\prime}$, with gelation seen at $2.9 \mathrm{~min}$ after voltage activation. At $0 \mathrm{~V}$ (control), the G5-DBA ${ }_{20}$ observes self-curing with a lag-time of $25 \pm 5$ min following aqueous reconstitution (Fig. 2c). Self-curing is found to be tunable with exposure to aerobic environments in a dry state, where oxygen exposure can increase ratios of catechol/quinone (Fig. S1, ESI $†$ ). Rapid initiation of crosslinking (fast increase of $G^{\prime}$ ) 
is observed at $-1 \mathrm{~V}$ for 15 and $30 \mathrm{wt} \%$ formulations (Fig. 2d). High solute (45 wt\%) reconstituted formulations are solid aqueous gels but dissolve in methanol (data not shown). When $+1 \mathrm{~V}$ is applied, a lag time of 2-3 min is noted before crosslinking with a retarded gelation time of $18 \mathrm{~min}$. The voltage polarity simply swaps the working electrode into a cathode $(-1 \mathrm{~V}$, acceptor reduction) or anode ( $+1 \mathrm{~V}$, donor oxidation), where the working/counter electrodes have a surface area ratio of $4 / 1$, respectively. In the catecholfree $\mathrm{G} 5-\mathrm{Benz}_{20}$ formulation, no crosslinking is observed at either polarity; therefore, crosslinking is solely mediated by the oxidation of catechol (Fig. S10, ESI $\dagger$ ). However, the Schiff base-free reduced $\mathrm{G} 5-\mathrm{DBA}_{20}$ loses the ability to be instantly reduced and also has a delayed gelation time of $8.4 \mathrm{~min} v s .3 \mathrm{~min}$. Taken together, these observations suggest that reduction of the Schiff base is the rate limiting reaction under the $-1 \mathrm{~V}$ conditions, whereby a cathode working electrode facilitates rapid initiation by providing a larger area. Oxygen mediated pathways are responsible for the self-curing behavior, and the $+1 \mathrm{~V}$ is likely a mixture of both mechanisms. However, minimum grafting of catechol is required before voltage mediated crosslinking is observed, as $\mathrm{G}^{-\mathrm{DBA}_{10}}$ in Fig. S10 (ESI $\dagger$ ) displays no gelation within $30 \mathrm{~min}$ but can be chemically cured. Fig. 2 d compares the $G^{\prime}$ values of all electrocuring formulations to two-part curing with periodate. G5-DBA 20 and $\mathrm{G}_{2}-\mathrm{DBA}_{30}$ activation by electrocuring or periodate displayed no significant differences after $60 \mathrm{~min}$.

Lap shear adhesion is evaluated with wet collagen films, which serve as a mimic of wet tissue substrates. ${ }^{28}$ G5-DBA $_{20}$ at $30 \mathrm{wt} \%$ is applied to the collagen and activated with disposable 3-electrode chips (Fig. 3). G5-DBA 20 formulation is cured by two methods: electrocuring $(-1 \mathrm{~V})$ vs. two-part curing (periodate). Electrocuring at $+1 \mathrm{~V}$ is not evaluated due to the inferior material properties and gelation time, as shown in the electrorheology analyses. Lap shear adhesion strength at failure evaluates the crosslinked matrix since cohesive failure is seen throughout.

Tack evaluation under 30 min demonstrates that electrocuring G5-DBA 20 formulation has a significant increase over two-part curing, and no adhesion is present for the self-curing formulation. The self-curing control sample (atmosphere exposure with neither voltage nor periodate applied) displays only viscous liquid material properties (Fig. 3b). The lap shear adhesion strength comparisons after $60 \mathrm{~min}$ are indicated in Fig. 3c. The self-curing formulation has an increase of adhesion strength to $\sim 4 \mathrm{~N} \mathrm{~cm}^{-2}$ (40 $\mathrm{kPa})$, which is about 4 times higher than $30 \mathrm{~min}$ case. Electrocuring continuously for $60 \mathrm{~min}$ is non-inferior to twopart curing, which both present $\sim 5 \mathrm{~N} \mathrm{~cm}^{-2}$ (50 kPa) adhesion strength. In summary, an azomethine/catechol adhesive is simply synthesized under ambient conditions to yield an acceptor/donor pair. The spontaneous reaction creates a redox-responsive adhesive that can be applied and activated by a number of external stimuli. The inclusion of both electron donor and acceptor groups creates an adhesive that can self-cure after a predictable lag-time. Activation can be triggered under both oxidative and reducing environments. For example, gelation time decreased in the following oxidative conditions: ambient atmosphere, $+1 \mathrm{~V}$, or periodate. A reducing environment triggered cross-linking instantaneously, $-1 \mathrm{~V}$ or aluminum substrates $\left(\mathrm{Al} \rightarrow \mathrm{Al}^{3+}+3 \mathrm{e}^{-}\right)$. a

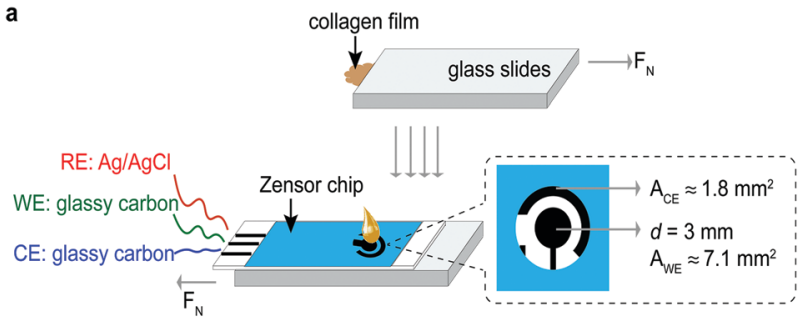

b

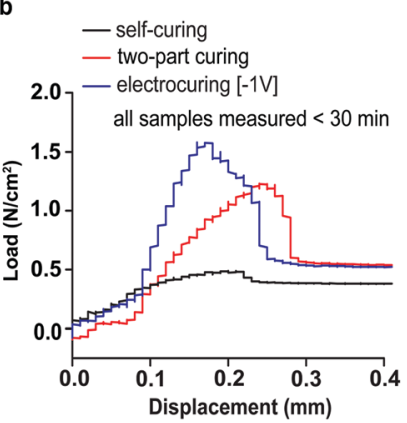

c

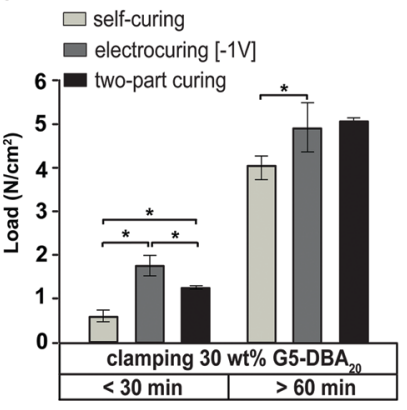

Fig. 3 Schematic illustration of: (a) electrocuring of $30 w t \%$ G5-DBA 20 formulation with the 3 -electrode chip and wet collagen film. Inset: Geometry and effective working area of electrodes on Zensor ${ }^{\circledR}$ chip. WE: working electrode, CE: counter electrode, RE: reference electrode. $F_{N}$ indicates the pulling force and directions. (b) Representative stress/strain plots of $30 \mathrm{wt} \% \mathrm{G} 5-\mathrm{DBA}_{20}$ formulations: self-curing, electrocuring $(-1 \mathrm{~V})$, and two-part curing (with $0.1 \mathrm{mM} \mathrm{NalO}_{4}$ ). (c) Lap shear adhesion strength of $30 \mathrm{wt} \% \mathrm{G} 5-\mathrm{DBA}_{20}$ formulation in comparison of self-curing, electrocuring, and two-part curing in $<30$ min and $>60$ min curing period.

A serendipitous finding was the short-term stability in aqueous solvents, but this allows development of self-curing adhesion that is semi-stable in a dry state. A bioadhesive that allows liquid manipulation and then self-cures with a known lag-time is a current unmet need for tissue repair. Activation of the lag-time 'clock' is as simple as aqueous reconstitution. The lag time of the dried and precipitated formulation drifted over a period of 3 months when exposed to aerobic environments. This suggests that the lag-time may be optimized in oxygen-free or limited exposure conditions (Fig. 2c). Future work will address tuning lag-time and further shelf-life stability.

For the first time, a catechol-adhesive allows activation by electrocuring, instead of traditional two-part curing method. This allows activation while avoiding side-effects from oxidation agents or highly concentrated metal chelators. Electrocuring adhesive strength was comparable to the periodate two-part curing methods. Two-part curing is a standard method of activation of mussel biomimetic adhesives, with modest adhesion strength $40 \mathrm{kPa}{ }^{8}$ Polydopamine-co-acrylate cured with periodate achieved up to $70 \mathrm{kPa}$ after one day of curing. Electrocuring allows a simpler approach with no additive mixing and more precise control over initiation and gelation time. However, formulations appear to be limited in design-successful electrocuring was only observed at $20-30 \%$ grafting ratios. Too little (10\%) grafting prevented voltage-activation-too much (greater than 40\%) displayed aqueous solubility problems that may stem from spontaneous crosslinking under storage. The electrocuring formulations herein have some advantages over our previous 
Voltaglue formulations. ${ }^{11,12,29}$ This redox donor/acceptor adhesives could be activated at $\pm 1 \mathrm{~V}$ ( $v s$. $\mathrm{Ag} / \mathrm{AgCl}$ ), but Voltaglue formulations need a higher voltage of $-1.6 \mathrm{~V}$ that evolves gases which can ultimately weaken the adhesive matrix and limit strength. However, Voltaglue appears more stable in oxidative and aqueous environments, as no spontaneous self-curing has been observed under ambient conditions.

To our surprise, voltage-activated crosslinking was accelerated when the working electrode was set as the cathode, effectively providing a larger surface area for reductive reactions. This supports the proposed hypothetical acceptor/donor mechanism in Fig. 1, but no direct evidence exists of Schiff base reduction. This is likely due to a combination of factors; G5-PAMAM contains both primary and tertiary amines, providing a local alkaline environment ( $\mathrm{pH}$ 9-10). If zwitterionic, the Schiff base catechol would attract electrons to the protonated azomethine (Fig. S1, $\mathrm{ESI} \dagger)$. At this high $\mathrm{pH}$, protons are limited to the zwitterion or other tautomers known to exist under aqueous conditions (see SI Results, ESI $\dagger$ ). A $-1 \mathrm{~V}$ electrochemical gradient at the working electrode attracts protons from atmosphere $\mathrm{O}_{2}$-mediated oxidation of catechol, which is thermodynamically favorable (but the internal electronic reduction/oxidation is not, see Fig. S2, ESI $\dagger$ ). With Schiff bases speculated to be reduced at this $-1 \mathrm{~V}$ potential, catechol to quinone oxidations are uninhibited. As quinones are responsible for crosslinking, their formation will decrease the time to gelation. Supporting this hypothesis is the empirical result that the aryl-Schiff bases are reduced with sodium borohydride (Fig. S8, ESI $\dagger$ ), where borohydride anion is estimated to have a formal potential of $E^{\circ \prime}=-0.7$ to $-0.43 \mathrm{~V}^{30}$

Many formulations of chitosan-catechol conjugates have been synthesized-chitosan is a cheap and scalable branched polymer that has been incorporated in many biomaterials. However, chitosan has an inherent solubility limit in acidic mediums that is often addressed with additional grafting. Even with catechol grafting, the functionalized chitosan can only achieve a $6 \mathrm{wt} \%$ solution (polymer in aqueous solvent)—which is too dilute for voltage-activation (G5-DBA 20 at $10 \mathrm{wt} \%$ displays no electrocuring). ${ }^{6,31}$ Grafting $19 \%$ to $80 \%$ of the total amines with catechol on chitosan displays similar material properties as the reported G5-DBA 20 formulations. ${ }^{15,32}$ Others have exploited electrochemical synthesis of chitosan-catechol, where reductive amination grafts chitosan to the surface followed by oxidative activation for catechol-chitosan grafting towards anti-oxidant surfaces. $^{33}$ The technology herein may allow a more straightforward approach for similar purposes. G4-PAMAM was grafted with PEG-catechol to form high modulus hydrogels up to $80 \mathrm{kPa},{ }^{34}$ but this required two-part curing with periodate and cure times of 48 hours. Future work will address methods to achieve similar high moduli with reversible, self-curing, and electrocuring properties while building on prior work to achieve in vivo implants and blood biocompatibility. ${ }^{35-37}$

\section{Conflicts of interest}

There are no conflicts to declare.

\section{Notes and references}

1 H. Lee, S. M. Dellatore, W. M. Miller and P. B. Messersmith, Science, 2007, 318, 426-430.

$2 \mathrm{~J} . \mathrm{Yu}, \mathrm{W}$. Wei, M. S. Menyo, A. Masic, J. H. Waite and J. N. Israelachvili, Biomacromolecules, 2013, 14, 1072-1077.

3 J. Y. Park, J. S. Kim and Y. S. Nam, Carbohydr. Polym., 2013, 97, 753-757.

4 M. Mehdizadeh, H. Weng, D. Gyawali, L. Tang and J. Yang, Biomaterials, 2012, 33, 7972-7983.

5 J. Yang, M. A. Cohen Stuart and M. Kamperman, Chem. Soc. Rev., 2014, 43, 8271-8298.

6 J. H. Ryu, S. Hong and H. Lee, Acta Biomater., 2015, 27, 101-115.

7 G. Westwood, T. N. Horton and J. J. Wilker, Macromolecules, 2007, 40, 3960-3964.

8 B. J. Kim, D. X. Oh, S. Kim, J. H. Seo, D. S. Hwang, A. Masic, D. K. Han and H. J. Cha, Biomacromolecules, 2014, 15, 1579-1585.

9 J. H. Ryu, Y. Lee, M. J. Do, S. D. Jo, J. S. Kim, B.-S. Kim, G.-I. Im, T. G. Park and H. Lee, Acta Biomater., 2014, 10, 224-233.

10 M. Cencer, Y. Liu, A. Winter, M. Murley, H. Meng and B. P. Lee, Biomacromolecules, 2014, 15, 2861-2869.

11 J. Ping, F. Gao, J. L. Chen, R. D. Webster and T. W. J. Steele, Nat. Commun., 2015, 6, 8050.

12 L. Gan, N. C. S. Tan, A. H. Shah, R. D. Webster, S. L. Gan and T. W. J. Steele, Macromolecules, 2018, 51, 6661-6672.

13 D. Nematollahi and S. Dehdashtian, Tetrahedron Lett., 2008, 49, 645-649.

14 J. H. Billman and A. C. Diesing, J. Org. Chem., 1957, 22, 1068-1070. 15 P. S. Yavvari and A. Srivastava, J. Mater. Chem. B, 2015, 3, 899-910. 16 H. S. Nanda, M. Singh and T. W. J. Steele, ECS Trans., 2017, 77, 547-555.

17 L. Gan, N. C. S. Tan and T. W. J. Steele, ECS Trans., 2017, 77, 981-988. 18 P. K. Maiti, T. Çağın, G. Wang and W. A. Goddard, Macromolecules, 2004, 37, 6236-6254.

19 C. Ornelas, Macromol. Chem. Phys., 2016, 217, 149-174.

20 A. J. Bard and L. R. Faulkner, Electrochemical Methods: Fundamentals and Applications, Wiley, 2000.

21 D. Nematollahi and S. M. Golabi, Electroanalysis, 2001, 13, 1008-1015.

22 M. F. Delbem, W. J. Baader and S. H. P. Serrano, Quim. Nova, 2002, $25,741-747$.

23 H. Ohmori, A. Matsumoto, M. Masui and H. Sayo, J. Electrochem. Soc., 1977, 124, 1849-1854.

24 M. Masui and H. Ohmori, J. Chem. Soc., Perkin Trans. 2, 1972, 1882-1887.

25 C. I. Simionescu, M. Grovu-Ivanoiu, I. Cianga, M. Grigoras, A. Duca and I. Cocârla, Angew. Makromol. Chem., 1996, 239, 1-12.

26 R. Das, A. Saxena, S. Saxena and G. Khan, J. Adv. Electrochem., 2015, 1, 19-24.

27 E. H. Cordes and W. P. Jencks, J. Am. Chem. Soc., 1963, 85, 2843-2848.

28 T. W. J. Steele, C. L. Huang, E. Nguyen, U. Sarig, S. Kumar, E. Widjaja, J. S. C. Loo, M. Machluf, F. Boey, Z. Vukadinovic, A. Hilfiker and S. S. Venkatraman, J. Mater. Sci.: Mater. Med., 2013, 24, 2013-2027.

29 M. Singh, H. S. Nanda, R. D. O'Rorke, A. E. Jakus, A. H. Shah, R. N. Shah, R. D. Webster and T. W. J. Steele, Adv. Healthcare Mater., 2018, 7, 1800538.

30 N. G. Connelly and W. E. Geiger, Chem. Rev., 1996, 96, 877-910.

31 K. Kim, J. H. Ryu, D. Y. Lee and H. Lee, Biomater. Sci., 2013, 1, 783-790.

$32 \mathrm{~J} . \mathrm{Xu}, \mathrm{S}$. Strandman, J. X. X. Zhu, J. Barralet and M. Cerruti, Biomaterials, 2015, 37, 395-404.

33 C. Cao, E. Kim, Y. Liu, M. Kang, J. Li, J.-J. Yin, H. Liu, X. Qu, C. Liu, W. E. Bentley and G. F. Payne, Biomacromolecules, 2018, 19, 3502-3514.

34 Y. Wang, Q. Zhao, Y. Luo, Z. Xu, H. Zhang, S. Yang, Y. Wei and X. Jia, Chem. Commun., 2015, 51, 16786-16789.

35 H. S. Nanda, A. H. Shah, G. Wicaksono, O. Pokholenko, F. Gao, I. Djordjevic and T. W. J. Steele, Biomacromolecules, 2018, 19, $1425-1434$

36 F. Gao, I. Djordjevic, O. Pokholenko, H. Zhang, J. Zhang and T. W. J. Steele, Molecules, 2018, 23, 796.

37 A. H. Shah, O. Pokholenko, H. S. Nanda and T. W. J. Steele, Mater. Sci. Eng., C, 2019, 100, 215-225. 